\title{
Raciocínio clínico do enfermeiro: uma abordagem segundo a Teoria do Processo Dual
}

\author{
Nurses' clinical reasoning: a Dual Process Theory approach \\ Razonamiento clínico del enfermero: un enfoque según la Teoría del Proceso Dual
}

\author{
Adrieli Quaresma'; Daiani Modernel Xavier"'; Marta Regina Cezar-Vaz"II
}

\begin{abstract}
RESUMO
Objetivo: refletir acerca do raciocínio clínico do enfermeiro na perspectiva da Teoria do Processo Dual. Conteúdo: trata-se de um estudo teórico-reflexivo construído, a partir da leitura crítica da Teoria do Processo Dual e de artigos científicos acerca do raciocínio clínico em enfermagem, buscados na Biblioteca Virtual em Saúde e Pubmed. O raciocínio clínico é composto por dois sistemas de processamento de informações que possibilitam a tomada de decisões em um contexto clínico. Constitui base para o desenvolvimento do processo de enfermagem e gerência do cuidado. Ademais, favorece identidade, visibilidade e confiabilidade da enfermagem como profissão da área da saúde, ao proporcionar a ruptura com a prática do cuidado mecanizado, tornando-o algo pensado e sustentado por conhecimentos científicos. Conclusão: este estudo permitiu a reflexão acerca do raciocínio clínico do enfermeiro na perspectiva da Teoria do Processo Dual. Espera-se subsidiar ações de desenvolvimento e aprimoramento dessa habilidade na assistência e no ensino.
\end{abstract}

Descritores: Habilidades de pensamento; tomada de decisão clínica; assistência de enfermagem; enfermagem

\section{ABSTRACT}

Objective: to think about nurses' clinical reasoning from the Dual Process Theory perspective. Content: this reflectivetheoretical study drew on a critical reading of Dual Process Theory and of scientific articles about clinical reasoning in nursing, found by searching the Virtual Health Library and Pubmed. The clinical reasoning comprises two information processing systems that enable decision making in a clinical context. It forms the basis for the nursing process and management of care. Moreover, it favors the identity, visibility, and reliability of nursing as a profession of the health field, as it fosters a break with the practice of mechanical care by making care something thoughtful and underpinned by scientific knowledge. Conclusion: this study made it possible to think, from the Dual Process Theory perspective, about nurses' clinical reasoning. It is hoped this will inform measures to develop and improve this skill in care and teaching.

Descriptors: Thinking; clinical decision-making; nursing care; nursing.

\section{RESUMEN}

Objetivo: reflexionar sobre el razonamiento clínico del enfermero en la perspectiva de la Teoría del Proceso Dual. Contenido: estudio teórico-reflexivo construido con base en la lectura crítica de la Teoría del Proceso Dual y de artículos científicos acerca del raciocinio clínico en enfermería, buscados en la Biblioteca Virtual en Salud y Pubmed. El razonamiento clínico se compone de dos sistemas de procesamiento de información que posibilitan la toma de decisiones en un contexto clínico. Constituye base para el desarrollo del proceso de enfermería y gestión del cuidado. Además, favorece la identidad, la visibilidad y la confiabilidad de la enfermería como profesión del área de la salud, al promover una ruptura con la práctica del cuidado mecanizado, haciéndolo algo pensado y sostenido por conocimientos científicos. Conclusión: este estudio permitió la reflexión acerca del razonamiento clínico del enfermero bajo la perspectiva de la Teoría del Proceso Dual. Se espera subsidiar acciones de desarrollo y perfeccionamiento de esa habilidad en la asistencia y enseñanza.

Descriptores: Pensamiento; toma de decisiones clínicas; atención de enfermería; enfermería.

\section{INTRODUÇÃO}

Atualmente, vive-se um período caracterizado por intensas modificações no trabalho em saúde, em decorrência da globalização, das transformações socioeconômicas e culturais, do avanço tecnológico e da incorporação de métodos mais avançados e complexos para promoção, prevenção, manutenção e recuperação da saúde. Isso resulta em mudanças no processo de cuidar, exigindo do enfermeiro não apenas técnicas precisas, mas, também, desenvolvimento, domínio e aprimoramento de habilidades cognitivas, como o raciocínio clínico ${ }^{1,2}$.

'Acadêmica do Curso de Graduação em Enfermagem, Escola de Enfermagem, Universidade Federal do Rio Grande.Brasil. E-mail: theadriquaresma@gmail.com "Doutora em Enfermagem. Professora Adjunta, Escola de Enfermagem, Universidade Federal do Rio Grande. Brasil. E-mail: daiamoder@gmail.com

I"Doutora em Filosofia da Enfermagem. Professora Titular, Escola de Enfermagem, Universidade Federal do Rio Grande. Brasil. E-mail: cezarvaz@vetorial.net 
O raciocínio clínico, considerado sinônimo de pensamento crítico ou julgamento clínico, pode ser entendido como um conjunto de processos cognitivos complexos que leva à tomada de decisões em um contexto clínico. Ele é fundamentado no saber científico e envolve julgamento intencional de informações, interpretação, análise, inferência de resultados e justificativa das decisões/ações ${ }^{3}$.

Nesse sentido, o enfermeiro faz uso do raciocínio clínico para coletar e interpretar os dados do histórico de saúde dos pacientes, verificar seu atual estado geral de saúde e suas necessidades, planejar e executar os cuidados necessários para atendê-las, bem como para avaliar sua efetividade. Para tal, torna-se importante o intercâmbio de informações entre enfermeiro e paciente, por meio da comunicação verbal e não verbal e das técnicas de avaliação clínica ${ }^{1}$.

Para que esse intercâmbio seja produtivo, o enfermeiro deve possuir e integrar conhecimentos de diversas áreas, como anatomia, fisiologia, fisiopatologia de doenças, semiologia e farmacologia, a fim de fundamentar o raciocínio clínico e, por conseguinte, assegurar ações de cuidado seguras e capazes de produzir resultados de saúde desejáveis ${ }^{4}$. Além disso, demanda, desse profissional, atitudes inquisitivas, compreensivas, reflexivas, criativas e perseverantes, de modo a garantir acesso às informações essenciais para a tomada de decisão terapêutica, reconhecer a singularidade de cada paciente e flexibilizar os cuidados de enfermagem, conforme suas necessidades 5 .

Os processos cognitivos que compõem o raciocínio clínico tornaram-se foco das pesquisas científicas em 1970. Nesse período, um psicólogo inglês ${ }^{6}$, que investigava a cognição humana, propôs e descreveu dois componentes de raciocínio: um rápido e indutivo e outro lento e dedutivo, originando a Teoria do Processo Dual. Essa teoria foi testada e validada por pesquisadores de diversas áreas do conhecimento ${ }^{7}$, inclusive da enfermagem ${ }^{8}$, sendo a mais aceita para a explicação dos processos cognitivos envolvidos no raciocínio clínico ${ }^{7-9}$. Na atualidade, um dos pesquisadores mais respeitados nos estudos referentes à Teoria do Processo Dual é o teórico israelense Daniel Kahneman. Em vista disso, o presente estudo abordará alguns aspectos da referida teoria, a partir do estudo mais recente de Kahneman ${ }^{9}$.

Partindo do pressuposto que o raciocínio clínico permeia as ações de cuidado e gerência do cuidar, exercidas pelo enfermeiro, justifica-se a reflexão e aprofundamento teórico acerca da temática, o que poderá subsidiar estratégias de aprimoramento dessa habilidade. Diante disso, objetivou-se refletir acerca do raciocínio clínico do enfermeiro numa perspectiva da Teoria do Processo Dual.

Trata-se de um estudo teórico-reflexivo, fundamentado na Teoria do Processo Dual $^{9}$ e sustentado pela literatura científica nacional e internacional. Com base na leitura crítica da referida teoria e de artigos a respeito do raciocínio clínico em enfermagem, os quais foram buscados na Biblioteca Virtual em Saúde e na Pubmed, elaborou-se o presente estudo organizado nos tópicos: estrutura do raciocínio clínico; raciocínio clínico na enfermagem; e desenvolvimento e aprimoramento do raciocínio clínico.

\section{Estrutura do Raciocínio Clínico}

A Teoria do Processo Dual propõe a estruturação do raciocínio clínico em dois componentes, sendo um nãoanalítico, o sistema 1, e outro analítico, o sistema $2^{9}$. Esses componentes possuem centro de processamento localizado no sistema nervoso central, em nível de região medial do córtex pré-frontal e dorsolateral do córtex pré-frontal, respectivamente. Essas regiões cerebrais são responsáveis pelas funções executivas, como: atenção, processamento de informações, planejamento de ações e tomada de decisões ${ }^{7}$.

O sistema 1, raciocínio clínico não-analítico ou intuitivo, está associado à percepção sensorial e ao reconhecimento rápido de padrões. Independe da inteligência do indivíduo e da memória de curto prazo. Ele é rápido e automático, requer pouco ou nenhum esforço mental e acontece de forma involuntária ${ }^{7,9,10}$.

O raciocínio clínico não-analítico baseia-se em habilidades inatas ao ser humano, como: capacidade de perceber o mundo ao redor, reconhecer objetos e orientar a atenção. Essas habilidades tornam-se mais ágeis e automáticas na medida em que são praticadas. Algumas delas são compartilhadas pelas pessoas em geral, outras se desenvolvem apenas a partir de treinamento ${ }^{7,9}$.

Em contraste, o sistema 2 ou raciocínio clínico analítico é eventual, consciente e lógico. Fundamenta-se no saber científico, portanto, é proporcional aos conhecimentos e à inteligência do indivíduo. Envolve habilidades cognitivas de julgamento, interpretação, análise, inferência de resultados e justificativa das decisões/ações, de acordo com o embasamento teórico utilizado para fundamentar o raciocínio ${ }^{7,9}$.

O desempenho do raciocínio analítico requer atenção/concentração e esforço mental. Portanto, o processamento de informações pelo sistema dois não funcionará, ou funcionará de forma inadequada, quando o sujeito que está raciocinando não está atento ao objeto de raciocínio ou está parcialmente atento. Sendo assim, duas ou mais atividades processadas pelo sistema 2 de raciocínio não podem ser realizadas, concomitantemente, pois induzem a falhas, diferentemente do sistema 1 que pode processar mais de uma situação ao mesmo tempo, desde que já tenham sido experienciadas em outros momentos ${ }^{9}$.

Além disso, o funcionamento do sistema 2 de raciocínio demanda gastos energéticos. Isso pode ser explicado pelos próprios processos cognitivos que despendem das altas taxas de adenosina trifosfato e glicose ${ }^{7,9}$. Estudo clínico norte- 
americano comprovou esse achado por meio de exames laboratoriais. Os pesquisadores demonstraram que o processamento de informações pelo sistema analítico foi mais ágil e preciso nos indivíduos que consumiram limonada à base de glicose do que no grupo controle. Ainda, os gastos energéticos que ocorrem durante a atividade do sistema 2 podem ser explicados pelas respostas fisiológicas, geradas nesse processo, como: dilatação das pupilas, tensão muscular, aumento da pressão arterial e da frequência cardíaca ${ }^{11}$.

Embora sejam diferentes no que se refere à performance, os sistemas 1 e 2 atuam simultaneamente e em conjunto. No entanto, o primeiro funciona, constantemente, em sua capacidade máxima de trabalho, enquanto que o segundo, normalmente, atua em modo de pouco esforço, com ativação esporádica de sua capacidade total de processamento ${ }^{9}$.

O sistema 1 processa os mais diversos conteúdos que se apresentam no dia a dia, gerando impressões, intuições, intenções e sentimentos para o sistema 2 que, por sua vez, analisa esse conteúdo. Quando analisado e aceito pelo sistema analítico, esse conteúdo é tido como verdade e torna-se, então, um padrão a ser reconhecido pelo sistema nãoanalítico em ocasiões futuras ${ }^{7,9}$.

Nesse sentido, o sistema analítico, normalmente funcionando com ativação parcial de sua capacidade de processamento, monitora as atividades do sistema não-analítico, a fim de identificar e corrigir possíveis erros ${ }^{9}$. Os erros gerados pelo sistema 1 relacionam-se, principalmente, ao risco de viés ao comparar situações distintas. Muitas vezes, o sistema 2 consegue barrar esses erros, corrigi-los e reeducar/reformular os padrões do sistema não-analítico. No entanto, quando isso não ocorre, acontecem equívocos na produção de pensamentos e tomada de decisões ${ }^{7,9}$.

Por outro lado, quando o sistema não-analítico apresenta dificuldades para processar determinado conteúdo, o sistema analítico é ativado em sua capacidade total de processamento. Nesse caso, o conteúdo é julgado, interpretado, analisado e, por fim, é realizada a tomada de decisão. O raciocínio analítico, ainda, propõe justificativas à decisão, fortalecendo sua validade ${ }^{9}$. Os erros relacionados a esse componente de raciocínio são menos frequentes e, quando ocorrem, estão associados à carência de conhecimentos e/ou desatenção do indivíduo durante o processo de raciocínio ${ }^{7,9,10}$.

\section{Raciocínio Clínico na Enfermagem}

No contexto da assistência de enfermagem, o raciocínio clínico é um elemento primordial ao enfermeiro. Ele confere identidade, visibilidade e confiabilidade a esse profissional no âmbito da equipe multiprofissional de saúde. Isso se deve ao fato de que a habilidade de raciocinar clinicamente promove a ruptura com a prática do cuidado mecanizado, tornando-o algo pensado e sustentado pelo saber científico, de modo a garantir uma assistência de qualidade, eficiente e segura ${ }^{2,3}$.

O raciocínio clínico constitui base para o desenvolvimento das cinco etapas do processo de enfermagem ${ }^{3}$, a saber: histórico enfermagem, diagnósticos de enfermagem, planejamento de enfermagem, implementação e avaliação de enfermagem, conforme mostra a figura 1. Sendo assim, constitui um importante instrumento de sistematização e organização do trabalho do enfermeiro ${ }^{12}$.

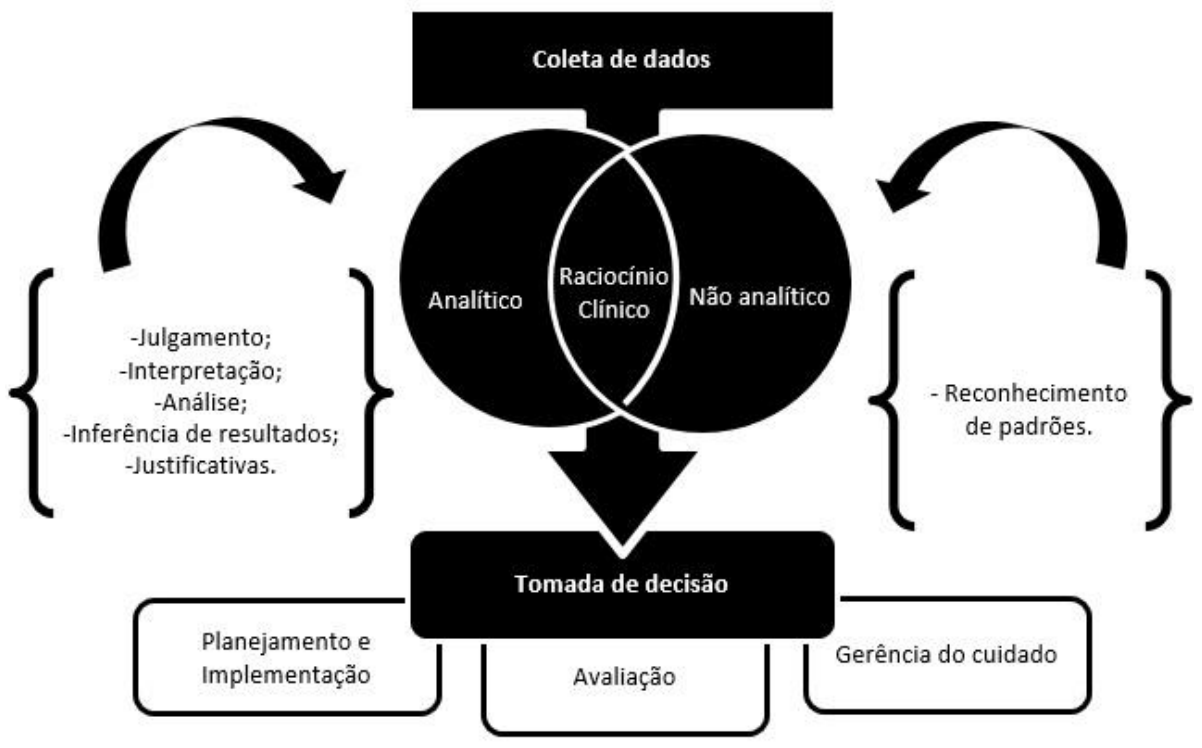

FIGURA 1: Diagrama representativo do raciocínio clínico aplicado à prática do enfermeiro de acordo com a Teoria do Processo Dual. Rio Grande, Rio Grande do Sul, Brasil, 2018. 
Na investigação do histórico de enfermagem, quando o enfermeiro avalia um indivíduo, identifica suas queixas, sinais e sintomas e reconhece-os como características de uma determinada condição clínica, é iniciado automaticamente o sistema 1 de raciocínio. Sendo assim, a identificação do estado geral do paciente, por meio dos diagnósticos de enfermagem, assim como a tomada de decisões acerca dos cuidados necessários em seu contexto clínico, por meio do planejamento de enfermagem e da implementação, ocorrerá de forma intuitiva, mediante reconhecimento de padrões decorrentes de experiências prévias do enfermeiro com situações clínicas semelhantes ${ }^{5,7,10}$.

Em contraponto, quando o enfermeiro identifica as queixas, sinais e sintomas do indivíduo, porém não os associa a uma determinada condição clínica, é ativado o sistema 2 de raciocínio. Nesse caso, o profissional avalia a história de saúde atual, pregressa e familiar do indivíduo, os achados do exame físico e dados complementares, como exames laboratoriais e de imagem (histórico de enfermagem), interpreta os dados subjetivos e objetivos coletados, a fim de determinar seus significados, analisa-os, de modo a identificar as necessidades do indivíduo (diagnósticos de enfermagem), e, por fim, infere os cuidados adequados às necessidades verificadas (planejamento de enfermagem e implementação). Além disso, justifica cada cuidado estabelecido, baseado em seus conhecimentos científicos e teorias da enfermagem ${ }^{3,5,10,13}$.

Após o desenvolvimento do histórico de enfermagem, estipulação dos diagnósticos de enfermagem, do planejamento enfermagem e da implementação dos cuidados, o enfermeiro avalia a assistência prestada, a evolução clínica do indivíduo e a efetividade ou não dos cuidados realizados. A partir disso, considera a necessidade ou não de readaptar as etapas anteriores do processo de enfermagem ${ }^{5,10}$. A etapa de avaliação realizada, geralmente, pelo sistema analítico, possibilita a identificação de falhas no processo de enfermagem e sua readaptação, bem como a reformulação dos padrões do sistema não-analítico de raciocínio.

Ademais, o raciocínio clínico é empregado pelo enfermeiro no gerenciamento do cuidado. Isso significa que ele, também, é utilizado para a tomada de decisões no que se refere à divisão do trabalho entre os membros da equipe de enfermagem, educação continuada dos profissionais e desenvolvimento e implementação de protocolos clínicos ${ }^{14}$. Portanto, o raciocínio clínico apresenta potencial para promover autonomia e desenvolvimento profissional do enfermeiro, aperfeiçoamento do processo de cuidar, qualidade e segurança no cuidado prestado ${ }^{15}$. A síntese do raciocínio clínico aplicado à prática do enfermeiro, conforme os pressupostos da Teoria do Processo Dual, é apresentada na Figura 1.

\section{Desenvolvimento e Aprimoramento do Raciocínio Clínico}

O raciocínio clínico se desenvolve num processo contínuo e inacabado, que tem início durante a Graduação em Enfermagem e vai aperfeiçoando-se ao decorrer da trajetória profissional do enfermeiro ${ }^{2}$. Algumas estratégias de ensinoaprendizagem podem contribuir nesse processo. São elas: estudos de caso, simulação clínica, questionamentos, mapas conceituais e aprendizagem interativa. No entanto, a solidificação do raciocínio clínico ocorre, principalmente, por meio de experiências vivenciadas na prática clínica ${ }^{13,16}$.

Nesse sentido, estudo iraniano verificou que enfermeiros mais experientes, com maior tempo de trabalho na área assistencial, utilizam, predominantemente, o raciocínio clínico não-analítico para a tomada de decisões, pois confiam em suas habilidades de reconhecer padrões. Atestou, ainda, que os enfermeiros recém-formados, valem-se do raciocínio clínico analítico, devido à insegurança e falta de experiências clínicas suficientes a ponto de permitir o reconhecimento de padrões ${ }^{17}$. Paralelamente, estudo australiano constatou que os profissionais com maior tempo de experiência clínica possuem raciocínio clínico acurado e ágil, enquanto que aqueles que têm pouco tempo de experiência apresentam dificuldades para coletar dados do histórico de saúde e integrá-los aos seus conhecimentos científicos, lentificando o processo de raciocínio clínico ${ }^{13}$.

Ao decorrer de sua trajetória assistencial, o enfermeiro vivencia e processa muitas informações clínicas semelhantes, as quais, geralmente, levam à determinação de cuidados parecidos ${ }^{17}$. Assim, pode-se inferir que 0 processamento repetido dessas informações pelo sistema 2 de raciocínio proporciona o reabastecimento dos padrões reconhecidos pelo sistema 1 , fazendo com que essas informações sejam rapidamente reconhecidas e processadas pelo sistema não-analítico, nas próximas vezes que forem vivenciadas. Isso pode justificar o porquê de os enfermeiros com maior tempo de experiência clínica utilizarem, predominantemente, o raciocínio clínico não-analítico ao invés do analítico e de os enfermeiros com pouca experiência, geralmente, usarem o raciocínio clínico analítico.

O modo pelo qual o enfermeiro aplica o raciocínio clínico durante a execução do processo de enfermagem sistema 1 ou sistema 2 - pode ser influenciado por seus conhecimentos técnico-científicos, sua habilidade de pensar/raciocinar, aspectos referentes ao seu ambiente de trabalho e seus valores, crenças e aspectos éticos e morais $^{16,18,19}$. Por isso, torna-se importante que o enfermeiro esteja constantemente atualizando seus conhecimentos e aperfeiçoando suas habilidades de raciocínio, por meio de técnicas de ensino-aprendizagem, como as já descritas neste texto. Também, é indispensável que, durante o processo de raciocínio clínico, esse profissional seja orientado por 
conhecimentos científicos, aspectos legais e éticos e pelas necessidades e metas de saúde dos pacientes, abandonando suas crenças e valores pessoais, de modo a garantir tomadas de decisões as mais adequadas e imparciais possíveis.

\section{CONCLUSÃO}

Este estudo permitiu refletir acerca do raciocínio clínico do enfermeiro na perspectiva da Teoria do Processo Dual. Destaca-se que o raciocínio clínico constitui um importante instrumento de trabalho para o enfermeiro, visto que fundamenta o processo de enfermagem e a gerência do cuidado. Além disso, favorece a identidade, visibilidade e confiabilidade da enfermagem como profissão da área da saúde.

Verificou-se que o raciocínio clínico envolve dois componentes de processamento de informações, por meio dos quais são desencadeados processos de reconhecimento de padrões, julgamento, interpretação, análise, inferência de resultados e justificativa das decisões tomadas, que imprimem validade e rigor científico à assistência de enfermagem. Evidenciaram-se, ainda, alguns fatores que podem interferir no processo de raciocínio clínico. São eles: conhecimentos técnico-científicos, habilidades de pensar/racionar, experiência clínica, valores, crenças e aspectos relativos ao ambiente de trabalho. Sendo assim, a compreensão da estrutura e funcionamento do raciocínio, conforme os pressupostos da Teoria do Processo Dual, assim como o conhecimento acerca dos fatores intervenientes nesse processo, tem potencial para favorecer técnicas de aperfeiçoamentos dessa habilidade e promover a redução de erros de raciocínio, que podem conduzir a danos relacionados com a assistência à saúde.

As buscas realizadas para o desenvolvimento desta reflexão permitiram identificar que as pesquisas científicas acerca do raciocínio clínico em enfermagem direcionam-se, na maioria dos casos, ao ensino de graduação. Nesse sentido, identificaram-se, como lacunas no conhecimento, as estratégias que o enfermeiro utiliza para aprimorar seu raciocínio clínico, as facilidades e dificuldades que enfrenta para desenvolver essa habilidade na prática assistencial e os aspectos relativos ao ambiente de trabalho que podem influenciar nesse processo. Sugerem-se novas pesquisas acerca do raciocínio clínico abordando essas lacunas.

Como contribuições para a enfermagem, que este estudo pode auxiliar no aprofundamento reflexivo acerca do raciocínio clínico sob a perspectiva da Teoria do Processo Dual. Espera-se, com isso, subsidiar ações de desenvolvimento e aprimoramento da habilidade de raciocínio clínico na prática do enfermeiro e no ensino em enfermagem, fazendo a interligação da pesquisa científica com as práticas pedagógicas e assistenciais.

\section{REFERÊNCIAS}

1. Danski MTR, Oliveira GLRD, Pedrolo E, Lind J, Johann DA. Importance of evidence-based practice in nurse's work processes. Ciênc. cuid. saúde. [Internet]. 2017 [cited 2018 Sep 8]; 16(2):1-6. Available from: http://www.periodicos.uem.br/ojs/index.php/CiencCuidSaude/article/view/36304/20831

2. Menezes SSCD, Corrêa CG, Gengo e Silva RDC, Da Cruz DDAML. Clinical reasoning in undergraduate nursing education: a scoping review. Esc. Enferm. USP. [Internet]. 2015 [cited 2018 Sep 8]; 49(6):1037-44. Available from: http://www.scielo.br/pdf/reeusp/v49n6/0080-6234-reeusp-49-06-1037.pdf

3. Lee J, Lee YJ, Bae J, Seo M. Registered nurses clinical reasoning skills and reasoning process: a think-aloud study. Nurse educ. today. [Internet]. 2016 [cited 2018 Sep 8]; 46:75-80. Available from: https://www.ncbi.nlm.nih.gov/pubmed/27611485

4. Busanello J, De Pinto DM, Chaves OCS, Schons EDS, Tonin D, Freire JM. Clinical evaluation in the academic practice of nurses: pleas to the nursing care. Rev. enferm. UFPE on line. [Internet]. 2016 [cited 2018 Sep 8]; 10(6):2279-2285. Available from: https://periodicos.ufpe.br/revistas/revistaenfermagem/article/view/11245/12854

5. Lunney M, Almeida MDA, Amiucci B, Aragazi IMM, De Barros ALBL, Bauman SL. et al. Pensamento crítico para alcance de resultados positivos em saúde: análises e estudos de caso em enfermagem. Porto Alegre (RS): Artmed; 2011.

6. Wason PC, Evans JSTBT. Dual processes in reasoning? Cognition [Internet]. 1974 [cited 2018 Sep 8]; 3(2):141-54. Available from: https://www.sciencedirect.com/science/article/pii/0010027774900171

7. Norman G. Dual processing and diagnostic errors. Adv. Health Sci. Educ. Theory Pract. [Internet]. 2009 [cited 2018 Sep 8]; 14(1):37-49. Available from: https://www.ncbi.nlm.nih.gov/pubmed/19669921

8. Paley J, Cheyne H, Dalgleish L, Ducan EAS, Niven CA. Nursing's ways of knowing and dual process theories of cognition. J. Adv. Nurs. [Internet]. 2007 [cited 2018 Sep 8]; 60(6):692-701. Available from: https://www.ncbi.nlm.nih.gov/pubmed/18039256

9. Kahneman D. Thinking, fast and slow. New York: Farrar, Straus and Giroux; 2013.

10. Yazdani S, Hosseinzadeh M, Hosseini F. Models of clinical reasoning with a focus on general practice: a critical review. J. Adv. Med. Educ. Prof. [Internet]. 2017 [cited 2018 Sep 12]; 5(4):177-84. Available from: https://www.ncbi.nlm.nih.gov/pmc/articles/PMC5611427/

11. Masicampo EJ, Baumeister RF. Toward a physiology of dual-process reasoning and judgment. Psychol. Sci. [Internet]. 2008 [cited 2018 Sep 12]; 19:255-60. Available from: https://www.ncbi.nlm.nih.gov/pubmed/18315798

12. Conselho Federal de Enfermagem (Br). Resolução 358, de 15 de outubro de 2009. Dispõe sobre a sistematização da assistência de enfermagem e a implementação do processo de enfermagem em ambientes públicos ou privados em que ocorre o cuidado 
profissional de enfermagem e dá outras providências [Internet]. 2009; [citado em 12 set 2018]. Disponível em: http://www.cofen.gov.br/resoluo-cofen-3582009_4384.html

13. Delany $\mathrm{C}$, Golding $\mathrm{C}$. Teaching clinical reasoning by making thinking visible: an action research project with allied health clinical educators. BMC med. educ. [Internet]. 2014 [cited 2018 Sep 12]; 14(20):1-10. Available from: https://www.ncbi.nlm.nih.gov/pmc/articles/PMC3912345/pdf/1472-6920-14-20.pdf

14. Jensen R, Guedes EDS, Leite MMJ. Informatics competencies essential to decision making in nursing management. Esc. Enferm. USP. [Internet]. 2016 [cited 2018 Sep 12]; 50(1):112-20. Available from: http://www.scielo.br/pdf/reeusp/v50n1/0080-6234reeusp-50-01-0112.pdf

15. Carvalho EC, Cruz DDALMD, Herdman TH. Contribution of standardized languages for knowledge production, clinical reasoning and clinical nursing practice. Rev. bras. enferm. [Internet]. 2013 [citado 2018 Sep 12]; 66(esp):134-41. Disponível em: http://www.scielo.br/pdf/reben/v66nspe/v66nspea17.pdf

16. Carvalho ECD, Oliveira-Kumakura ARDS, Morais SCRV. Clinical reasoning in nursing: teaching strategies and assessment tools. Rev. bras. enferm. [Internet]. 2017 [cited 2018 Sep 18]; 70(3):690-6. Available from: http://www.scielo.br/pdf/reben/v70n3/0034-7167-reben-70-03-0662.pdf

17. Seidi J, Alhani F, Salsali M. Nurses clinical judgment development: a qualitative research in Iran. Iran. Red. Crescent. Med. J. [Internet]. 2015 [cited 2018 Sep 18]; 17(8):1-8. DOI: https://doi.org/10.5812/ircmj.20596

18. Dias JAA, David HMSL, Rodrigues BMRD, Peres PLP, Pacheco STDA, Oliveira MSD. Morality and critical thinking: essential competences in nurses' training. Rev. enferm. UERJ [Internet]. 2017 [cited 2018 Set 18]; 25:1-5. DOI: https://doi.org/10.12957/reuerj.2017.26391

19. Dias JAA, David HMSL, Acioli S, Santos RDSS, Santos FPDA. Critical thinking as a competence for nurses' practice in the family health strategy. Rev. enferm. UERJ [Internet]. 2018 [cited 2018 Set 18]; 26:1-5. DOI: https://doi.org/10.12957/reuerj.2018.30505 